\title{
A comparative analysis of the complete chloroplast genome sequences of four peanut botanical varieties
}

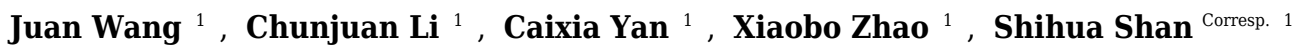 \\ ${ }^{1}$ Laboratory of Genetics and Breeding, Shandong Peanut Research Institute, Qingdao, Shandong, China \\ Corresponding Author: Shihua Shan \\ Email address: shansh1971@163.com
}

Background: Arachis hypogaea L. is an economically important oilseed crop worldwide comprising six botanical varieties. In this work, we characterized the chloroplast (cp) genome sequences of the four widely distributed peanut varieties.

Methods: The cp genome data of these four botanical varieties (var. hypogaea, var. hirsuta, var. fastigiata and var. vulgaris) were obtained by next-generation sequencing (NGS). These high throughput sequencing reads were then assembled, annotated and comparatively analyzed.

Results: The total $\mathrm{cp}$ genome lengths of the studied A. hypogaea varieties were 156,354 bp (var. hypogaea), 156,878 bp (var. hirsuta), 156,718 bp (var. fastigiata) and 156,399 bp (var. vulgaris). Comparative analysis of theses cp genome sequences revealed that their gene content, gene order and GC content were highly conserved, with only a total of 46 SNPs and 26 InDels identified. Most of the variation is restricted to non-coding sequences, especially, the trnl-GAU intron region was detected to be highly variable and will be useful for future evolutionary studies.

Discussion: The four $\mathrm{cp}$ genome sequences acquired here will provide valuable genetic resources for distinguishing $A$. hypogaea botanical varieties and determining their evolutionary relationship. 
1 A comparative analysis of the complete chloroplast genome sequences of four

2

3

4

5

6

$7 \quad{ }^{1}$ Laboratory of Genetics and Breeding, Shandong Peanut Research Institute, Qingdao, China

8

*Juan Wang and Chunjuan Li have contributed equally to this work.

10

$11{ }^{\#}$ corresponding author:

12 Shihua Shan

13

14 E-mail: shansh1971@163.com

\section{peanut botanical varieties}

Juan Wang ${ }^{1 *}$, Chunjuan $\mathrm{Li}^{1 *}$, Caixia Yan ${ }^{1}$, Xiaobo Zhao ${ }^{1}$, Shihua Shan ${ }^{1 \#}$ 
15

\section{Abstract}

Background: Arachis hypogaea L. is an economically important oilseed crop worldwide comprising six botanical varieties. In this work, we characterized the chloroplast (cp) genome sequences of the four widely distributed peanut varieties.

Methods: The cp genome data of these four botanical varieties (var. hypogaea, var. hirsuta, var. fastigiata and var. vulgaris) were obtained by next-generation sequencing (NGS). These high throughput sequencing reads were then assembled, annotated and comparatively analyzed.

Results: The total cp genome lengths of the studied A. hypogaea varieties were 156,354 bp (var. hypogaea), 156,878 bp (var. hirsuta), 156,718 bp (var. fastigiata) and 156,399 bp (var. vulgaris). Comparative analysis of theses cp genome sequences revealed that their gene content, gene order and GC content were highly conserved, with only a total of 46 SNPs and 26 InDels identified. Most of the variation is restricted to non-coding sequences, especially, the trnI-GAU intron region was detected to be highly variable and will be useful for future evolutionary studies.

Discussion: The four $\mathrm{cp}$ genome sequences acquired here will provide valuable genetic resources for distinguishing A. hypogaea botanical varieties and determining their evolutionary relationship.

\section{Short title:}

Peanut cp genome analysis

\section{Introduction}

Cultivated peanut (Arachis hypogaea L.) is one of the most important oilseed crops that is mainly planted in China, India, the United States of America and Argentina (Hammons 1994; Grabiele et al. 2012; Bertioli et al. 2016). Based on morphological (Gibbons et al. 1972; Krapovickas and 
Vanni 1960; 2010) and molecular (Gepts 1993; He and Prakash 2001) evidences, six botanical varieties of A. hypogaea have been identified: var. hypogaea, var. hirsuta, var. fastigiata, var. vulgaris (Gibbons et al. 1972), as well as var. aequatoriana and var. peruviana with the last two being region specific.

In land plants, the cp genome is circular and has a large single copy (LSC) region and a small single copy (SSC) region that are separated by a pair of inverted repeat (IR) regions (Raubeson and Jansen 2005). The major role of the chloroplast is to conduct photosynthesis; additionally, it is involved in the biosynthesis of fatty acids, vitamins, pigments and amino acids (Prabhudas et al. 2016). Different from nuclear sequence, the cp DNA has several advantages, including lowrecombination, haploid ploidy, and maternal inheritance, making cp DNA an ideal tool for evolutionary studies (Birky 2001; Wu and Ge 2012). For example, with the help of genetic markers that includes non-coding cpDNA regions (trnTR-trnS and $\operatorname{trn} T$-trnY), Grabiele et al. (2012) found that the six peanut botanical varieties were very likely to have a single genetic origin, however, the fine evolutionary relationship between these varieties remains to be resolved.

The rapid progress of high-throughput sequencing technology development has greatly facilitated the acquisition of cp genome data, which are not only powerful for reconstructing interspecific phylogeny (Jansen et al. 2007; Parks et al. 2009; Moore et al. 2010), but are also helpful for investigating genome dynamic at the subspecies level. For instance, Zhao et al. (2015) compared the cp genomes of four Chinese Panax ginseng strains and suggested that their genome dynamic was under selective pressure.

Although there are six botanical varieties within A. hypogaea that differ at both the morphological and molecular levels (Ferguson et al. 2004), only very limited A. hypogaea cp genome data are currently available (Prabhudas et al. 2016; Choi and Choi 2017). Here, we acquired and examined the complete cp genome nucleotide sequences of the four main peanut botanical varieties, providing valuable genetic resources for further evolutionary studies. 
64

65

66

67

68

\section{Materials and Methods}

DNA extraction and sequencing

Four representative A. hypogaea varieties (var. hypogaea, var. hirsuta, var. fastigiata and var. vulgaris) were collected from Shandong Peanut Research Institute, Qingdao, China. China has become the largest producer of cultivated peanut in the world ( $\mathrm{Yu}$ 2008), and these four main botanical varieties have been cultivated in China for more than 500 years. The seedlings were grown using hydroponic methods. The cp DNA was isolated from fresh leaves ( $>5 \mathrm{~g})$ of 3- to 4week-old seedlings using the Plant Chloroplast DNAOUT Kit (Bjbalb, China). The quality of $\mathrm{cp}$ DNA samples was checked by agarose gel electrophoresis with Super GelRed (US Everbright Inc, Suzhou, China). Libraries with an average length of $350 \mathrm{bp}$ were constructed using the NexteraXT DNA Library Preparation Kit (Illumina, China). The quality of the libraries was checked by GeneRead DNA QuantiMIZE Assay Kit (QIAGEN, Germany). Sequencing was performed on the Illumina HiSeq Xten platform (Illumina, China), and the average length of the generated reads was $150 \mathrm{bp}$.

\section{Data assembly and annotation}

The quality of the raw paired-end reads was assessed by FastQC v0.11.3 (Andrews 2014). All raw data for four A. hypogaea varieties were filtered based on the following rules: 1) adapter trimming; 2) quality control; each read has $<5 \%$ unidentified nucleotides and $>50 \%$ of its bases with a quality value of $>20$. This filtration was carried out using Cutadapt v1.7.1 (Martin 2011). The high-quality data were then assembled into contigs using the de novo assembler SPAdes v3.9.0 (Nurk et al. 2013), and these contigs were further assembled into complete cp genome using NOVOPlasty (Dierckxsens et al. 2016). The assembled data were checked against the published complete cp genome of A. hypogaea (GenBank accession no. KX257487, Prabhudas et al. 2016). The cp genes were annotated using the DOGMA tool with default parameters (Wyman et al. 2004). The cp genome images were drawn with OGDraw v1.2 (Lohse et al. 2007). 
91 Variation detection and evolutionary relationship analysis

92 Multiple sequence alignment was generated using VISTA and Mauve v2.3.1 software (Frazer et

93 al. 2004; Darling et al. 2010) and was checked manually when necessary. All alignments were 94 visualized using the VISTA viewer program (Mayor et al. 2000). Single nucleotide polymorphisms 95 (SNPs) were identified by Mauve v2.3.1. The insertions/deletions (InDels) were retrieved from the 96 sequence alignments using the mVISTA package. An InDels image including 10 bp up- and 97 downstream was then generated. Simple sequence repeats (SSRs) were isolated from all filtered 98 InDels. Repeat sequences with repeating units of 2-6 bp that repeated no fewer than three times 99 were considered as SSRs.

100 The genetic relationship of the four peanut $\mathrm{cp}$ genomes together with two available peanut $\mathrm{cp}$ 101 genome sequences (GenBank accession no. KX257487 and KJ468094; Prabhudas et al. 2016; 102 Choi and Choi 2017) were examined by constructing a minimum evolutionary (ME) tree using 103 MEGA v6 with default parameters (Tamura et al. 2011). The cp genome sequences from four other 104 related species (Robinia pseudoacacia, Ceratonia siliqua, Leucaena trichandra and Senna tora) 105 of Fabaceae were used as outgroups (CSI-BLAST E-value $<10^{-6}$ ).

\section{Results}

Assembly and validation of cp genomes

More than $1 \mathrm{~Gb}$ raw sequencing data per sample was generated from high-throughput sequencing. After cleaning and trimming, 22,511,400 (var. vulgaris) to 62,087,400 (var. hirsuta) paired-end reads were acquired, which were then mapped separately to the reference cp genome, attaining coverage amounts of $143 \times$ to $396 \times$. After performing de novo and reference-guided assembly with minor modifications, we acquired four complete cp genome sequences for A. hypogaea var. 
114 hypogaea, var. hirsuta, var. fastigiata and var. vulgaris (Figure 1; Table 1).

115 For each of the assembled cp genome sequences, a .sqn file that was generated by the Sequin 116 software (https://www.ncbi.nlm.nih.gov/projects/Sequin/), submitted to GenBank and acquired 117 the following accession numbers: MG814006 for var. fastigiata, MG814007 for var. hirsuta, 118 MG814008 for var. hypogaea and MG814009 for var. vulgaris. Users can download the data for 119 research purposes only when referencing this paper.

Genetic structure of the peanut cp genome

122 These four acquired peanut cp genomes were found to have the classical quadripartite structure of 123 land plant chloroplast genomes that comprises a LSC, a SSC and two IR (A/B) regions. The

124

125

126

127

128

129

130

131

132

133

134

135

136

137 sequence lengths among the four cp genomes ranged from 156,354 bp to $156,878 \mathrm{bp}$. The size varied from 85,900 bp (var. hirsuta) to 86,196 bp (var. fastigiata) in the LSC region, from 18,796 bp (var. hypogaea, var. hirsuta and var. vulgaris) to 18,874 bp (var. fastigiata) in the SSC region and from 25,806 bp (var. hypogaea) to 26,091 bp (var. hirsuta) in the IR (A/B) region (Table 1). A total of 110 genes were identified from the cp genome: four ribosomal RNA (rRNA) genes, 76 protein-coding genes and 30 transfer RNA (tRNA) genes (Table 2). Among the 110 identified genes, six protein-coding genes, six tRNA genes and four rRNA genes were distributed in the IR (A/B) regions. The cp genome consisted of $55.66 \%$ coding regions and $44.34 \%$ non-coding regions including both intergenic spacers and introns. The overall GC content of the cp genomic sequences was 36.3 36.4\%, and the GC contents of the LSC, SSC, and IR (A/B) regions were 33.8\%, 30.2 30.3\%, and 42.8 42.9\%, respectively (Table 2).

Variation among the cp genomes

Among the four acquired peanut $\mathrm{cp}$ genome sequences, there was no difference at the junction 
138

139

140

141

142

143

144

145

146

147

148

149

150

151

152

153

154

155

156

157

158

159

160

161

162

4

positions (Figure 2). A total of 46 SNPs were found within the quadripartite structural region. VISTA-based identity plots illustrated the hotspot regions of genetic variation among the cp genomes (Figure 3). As expected, non-coding sequences exhibited more variation than did the coding sequences, and greater amounts of substitutions were found in the trnI-GAU intron (25 SNPs) and the $y c f 3-p s a A$ spacer (8 SNPs) regions. The only identified non-synonymous was located within the psaA gene. The hydrophobic amino acid Tyr in var. hypogaea, var. fastigiata and var. vulgaris was replaced by the hydrophilic amino acid Asn in var. hirsuta.

A total of 26 InDels were identified: thirteen were located in spacers, nine were in introns, and four were in genes; 15 were in the LSC region, two were in the SSC region, and nine were in IR (A /B) regions (Supplementary Figure S1). Among these InDels, large InDels (> 50 bp) were found in the $p s k$-trn $Q$ intergenic spacer, the $\operatorname{trn} L$ intron and $y c f 1$. Among those InDels, we identified six SSR regions (sequence identity $>90 \%$ ): four A stretches and one T stretches ranging from 7 bp to $16 \mathrm{bp}$, as well as one with a CTAG repeat motif. No $\mathrm{C}$ or $\mathrm{G}$ stretches were identified. Moreover, InDels in the $y c f 1$ and the $y c f 2$ regions represent frameshift mutations: the $63 \mathrm{bp}-$ insertion at the end of the $y c f l$ gene led to a longer amino acid sequence in var. fastigiata, while a $18 \mathrm{bp}$-deletion was found in the middle of IR (A/B) ycf2 gene regions in var. hypogaea.

5 Genetic relationship analysis

6

Due to low genetic diversity, the whole cp genome sequences were used to construct an evolutionary tree based on ME algorithms. The results showed that these peanut cp genomes clustered into a monophyletic branch, while the four outgroup species were clustered into another branch. Among the six analyzed peanut cp genomes, var. hirsuta is relatively different from the rest and constitute a basal clade (Figure 4). The high support values (>99\%) were shown above the nodes. 
163

164

165

166

167

168

169

170

171

172

173

174

175

176

177

178

179

180

181

182

183

184

185

186

187

188

\section{Discussion}

The chloroplast (cp) is an important plant cell organelle (Alberts et al. 2002). The cp genome usually lacks recombination and are is maternally inherited and is therefore very useful for distinguishing taxa and inferring evolutionary relationships. Here, we have studied the cp genomes of cultivated peanut (A. hypogaea) that is an economically important oilseed crop worldwide. $A$. hypogaea comprised six varieties that differ at both the morphological and molecular levels (Ferguson et al. 2004). So far, only very limited A. hypogaea cp genome data are available (Prabhudas et al. 2016).

In the present study, we acquired and closely examined the whole $\mathrm{cp}$ genome sequences of four main peanut varieties. We found that the overall $\mathrm{cp}$ genome structures of the four botanical varieties were the same and displayed the classical quadripartite structure of land plant cp genome (Raubeson and Jansen 2005). No definitive genomic rearrangements or gene inversions were found among the four peanut $\mathrm{cp}$ genomes. The sequence variation among the four peanut $\mathrm{cp}$ genomes was also relatively limited, and most of them were restricted to the non-coding regions, especially the trnI-GAU intron exhibited an outstanding level of variation ( 25 out of the entire 46 identified SNPs), suggesting that the rapidly evolving nature of this intron. This trnI-GAU intron has therefore a great potential for developing molecular markers that could be used in future phylogenetic studies.

In addition, a minimum-evolution tree of the four acquired peanut $\mathrm{cp}$ genomes together with two earlier published peanut $\mathrm{cp}$ genomes has been constructed to speculate their evolutionary relationships. Our result showed that the six investigated peanut cp genomes form a monophyletic branch, and this agrees with earlier studies (Grabiele et al. 2012). In addition, our result also revealed that among the six studied peanut cp genomes, var. hirsuta was relatively more distantly related to the others and may constitute a basal branch, which was in line with the previous reports (Duan et al. 1995; Ferguson et al. 2004). Consistent with its suggested relationship between var. hirsuta and the other studied peanut varieties, var. hirsuta appeared to be the peanut variety found 
189 190

191

192

193

194

195

within the archeological remains along the Pacific coast of Perú (Bonavia) that may be the region of origin of cultivated peanut (Simpson et al 2001; Stalker et al. 2017).

\section{Conclusion}

With the help of high-throughput sequencing technology, we revealed the complete cp genomes of four main peanut botanical varieties. The gene contents and gene orders of the cp genomes were highly conserved. The trnI-GAU intron region was considered to be rapid-evolving region that could potentially serve as molecular markers in phylogenetic studies. This study will provide valuable cp genomic resources for future exploitation.

\section{Acknowledgements}

The authors would like to acknowledge Dr. Yuan Li from Lund University of Sweden and Dr. Dachuan Shi from Qingdao Academy of Agricultural Science of China for their excellent advices on this paper.

\section{References}

Alberts B, Johnson A, Lewis J, Raff M, Roberts K, and Watson JD. 2002. Molecular Biology of the Cell. 4th edition. Chapter 14: Chloroplasts and photosynthesis. New York: Garland Science.

Andrews S. 2014. FastQC: a quality control tool for high throughput sequence data. https://doi.org/citeulike-article-id:11583827

Bertioli DJ, Cannon SB, Froenicke L, Huang G, Farmer AD, Cannon EK, Liu X, Gao D, Clevenger J, Dash S, Ren L, Moretzsohn MC, Shirasawa K, Huang W, Vidigal B, Abernathy B, Chu 
212

213

214

215

216

217

218

219

220

221

222

223

224

225

226

227

228

229

230

231

232

233

234

235

Y, Niederhuth CE, Umale P, Araujo AC, Kozik A, Kim KD, Burow MD, Varshney RK, Wang X, Zhang X, Barkley N, Guimaraes PM, Isobe S, Guo B, Liao B, Stalker HT, Schmitz RJ, Scheffler BE, Leal-Bertioli SC, Xun X, Jackson SA, Michelmore R, and Ozias-Akins P. 2016. The genome sequences of Arachis duranensis and Arachis ipaensis, the diploid ancestors of cultivated peanut. Nature Genetics 48:438-446. 10.1038/ng.3517

Birky CW. 2001. The inheritance of genes in mitochondria and chloroplasts: laws, mechanisms, and models. Annual Review of Genetics 35:125-148.

Choi IS, and Choi BH. 2017. The distinct plastid genome structure of Maackia fauriei (Fabaceae: Papilionoideae) and its systematic implications for genistoids and tribe Sophoreae. PLoS One 12(4):e0173766. doi:10.1371/journal.pone.0173766

Darling AE, Mau B, and Perna NT. 2010. Progressive Mauve: multiple genome alignment with gene gain, loss and rearrangement. PLoS One 5:e11147. 10.1371/journal.pone.0011147

Dierckxsens N, Mardulyn P, and Smits G. 2017. NOVOPlasty: de novo assembly of organelle genomes from whole genome data. Nucleic Acids Research 45: e18. 10.1093/nar/gkw955

Duan NX, Jiang HF, Liao BS, and Zhou R. 1995. var. hirsuta in China: the origin and spread (in Chinese). Chinese Journal of Oil Crop Sciences 17 (2): 68-71.

Ferguson ME, Bramel PJ, and Chandra S. 2004. Gene Diversity among Botanical Varieties in Peanut (L.). Crop Science 44 (5): 1847-1854.

Frazer KA, Pachter L, Poliakov A, Rubin EM, and Dubchak I. 2004. VISTA: computational tools for comparative genomics. Nucleic Acids Research 32:W273-279. 10.1093/nar/gkh458

Gepts P. 1993. The use of molecular and biochemical markers in crop evolution studies. Evolutionary Biology-New York 27:51-94.

Gibbons RW, Bunting AH, and Smartt J. 1972. The classification of varieties of groundnut (Arachis hypogaea L.). Euphytica 21(1):78-85. 
236 Grabiele M, Chalup L, Robledo G, and Seijo G. 2012. Genetic and geographic origin of

237

238

239

240

241

242

243

244

245

246

247

248

249

250

251

252

253

254

255

256

257

258

259 domesticated peanut as evidenced by $5 \mathrm{~S}$ rDNA and chloroplast DNA sequences. Plant Systematics and Evolution 298:1151-1165.

Hammous RO. 1994. The origin and history of the groundnut. In: The groundnut crop: a scientific basis for improvement. Chapman and Hall, New York. 10.1007/978-94-011-0733-4_2

He G, and Prakash C. 2001. Evaluation of genetic relationships among botanical varieties of cultivated peanut (Arachis hypogaea L.) using AFLP markers. Genetic Resources and Crop Evolution 48:347-352. 10.1023/a:1012019600318

Jansen RK, Cai Z, Raubeson LA, Daniell H, Depamphilis CW, Leebens-Mack J, Muller KF, Guisinger-Bellian M, Haberle RC, Hansen AK, Chumley TW, Lee SB, Peery R, McNeal JR, Kuehl JV, and Boore JL. 2007. Analysis of 81 genes from 64 plastid genomes resolves relationships in angiosperms and identifies genome-scale evolutionary patterns. Proceedings of the National Academy of Sciences of the United States of America 104:19369-19374. 10.1073/pnas.0709121104

Krapovickas A, and Rigoni VA. 1960. La nomenclatura de las subespecies y variedades de Arachis hypogaea L. Revista De Investigaciones Agricolas "Alberto Boerger" 14: 198-228.

Krapovickas A, and Gregory WC. 1994. Taxonomia del énero Arachis (Leguminosae). Bonplandia 8: 1-186. 10.2307/41941177.

Krapovickas A, and Gregory WC. 2007. Taxonomy of the genus Arachis (Leguminosae). Bonplandia. 16: 7-205.

Lohse M, Drechsel O, and Bock R. 2007. OrganellarGenomeDRAW (OGDRAW): a tool for the easy generation of high-quality custom graphical maps of plastid and mitochondrial genomes. Current Genetics 52:267-274. 10.1007/s00294-007-0161-y

Katoh K, and Standley DM. 2013. MAFFT multiple sequence alignment software version 7: 
260

261

262

263

264

265

266

267

268

269

270

271

272

273

274

275

276

277

278

279

280

281

282

283

improvements in performance and usability. Molecular Biology and Evolution. 30(4):772780.

Martin M. 2011. Cutadapt removes adapter sequences from high-throughput sequencing reads. Embnet Journal 17(1). 10.14806/ej.17.1.200 pp. 10-12

Mayor C, Brudno M, Schwartz JR, Poliakov A, Rubin EM, Frazer KA, Pachter LS, and Dubchak I. 2000. VISTA: visualizing global DNA sequence alignments of arbitrary length. Bioinformatics 16:1046-1047.

Moore MJ, Soltis PS, Bell CD, Burleigh JG, and Soltis DE. 2010. Phylogenetic analysis of 83 plastid genes further resolves the early diversification of eudicots. Proceedings of the National Academy of Sciences of the United States of America 107:4623-4628. 10.1073/pnas.0907801107

Nurk S, Bankevich A, Antipov D, Gurevich A, Korobeynikov A, Lapidus A, Prjibelsky A, Pyshkin A, Sirotkin A, Sirotkin Y, Stepanauskas R, McLean J, Lasken R, Clingenpeel SR, Woyke T, Tesler G, Alekseyev MA, and Pevzner PA. 2013. Assembling genomes and minimetagenomes from highly chimeric reads. International Conference on Research in Computational Molecular Biology (Vol. 7821, pp.158-170). Springer, Berlin, Heidelberg.

Ohyama K, Fukuzawa H, Kohchi T, Shirai H, Sano T, Sano S, Umesono K, Shiki Y, Takeuchi M, and Chang Z. 1986. Chloroplast gene organization deduced from complete sequence of liverwort Marchantia polymorpha chloroplast DNA. Nature 322:572-574.

Okonechnikov K, Golosova O, and Fursov M, the UGENE team. 2012. Unipro UGENE: a unified bioinformatics toolkit. Bioinformatics 28: 1166-1167. 10.1093/bioinformatics/bts091

Parks M, Cronn R, and Liston A. 2009. Increasing phylogenetic resolution at low taxonomic levels using massively parallel sequencing of chloroplast genomes. BMC Biology 7:84-84.

Prabhudas SK, Prayaga S, Madasamy P, and Natarajan P. 2016. Shallow whole genome 
284

285

286

287

288

289

290

291

292

293

294

295

296

297

298

299

300

301

302

303

304

305

306

307

sequencing for the assembly of complete chloroplast genome sequence of Arachis hypogaea L. Frontier in Plant Science 7:1106. 10.3389/fpls.2016.01106

Raubeson LA, and Jansen RK. 2005. Chloroplast genomes of plants," in Plant diversity and evolution: genotypic and phenotypic variation in higher plants, ed Henry RJ. (Wallingford, CT: CABI), 45-68.

Shinozaki K, Ohme M, Tanaka M, Wakasugi T, Hayshida N, Matsubayasha T, Zaita N, Chunwongse J, Obokata J, and Yamaguchishinozaki K. 1986. The complete nucleotide sequence of the tobacco chloroplast genome. Plant Molecular Biology Reporter 4:111-148.

Simpson CE. 2001. Use of wild Arachis species/ introgression of genes in to A. hypogaea L. Peanut Science 28(2): 114-116.

Stalker HT. 2017. Utilizing wild species for peanut improvement. Crop Science 57(3): 1102-1120. 10.2135/cropsci2016.09.0824

Tamura K, Peterson D, Peterson N, Stecher G, Nei M, and Kumar S. 2011. MEGA5: molecular evolutionary genetics analysis using maximum likelihood, evolutionary distance, and maximum parsimony methods. Molecular Biology and Evolution 28:2731-2739. 10.1093/molbev/msr121

Wyman SK, Jansen RK, and Boore JL. 2004. Automatic annotation of organellar genomes with DOGMA. Bioinformatics 20:3252-3255. 10.1093/bioinformatics/bth352

Wu ZQ, and Ge S. 2012. Phylogeny of the BEP clade in grasses revisited: evidence from whole genome sequences of chloroplast. Molecular Phylogenetics and Evolution 62: 578-578. 10.1016/j.ympev.2011.10.019

Yin D, Wang Y, Zhang X, Ma X, He X, and Zhang J. 2017. Development of chloroplast genome resources for peanut (Arachis hypogaea L.) and other species of Arachis. Scientific Reports 7:11649. 10.1038/s41598-017-12026-xYu SL. 2008. Peanut varieties and their genealogy 
in China. Shanghai Science and Technology Press.

309 Zhao Y, Yin J, Guo H, Zhang Y, Xiao W, Sun CM, Wu J, Qu X, Yu J, and Wang X. 2015. The complete chloroplast genome provides insight into the evolution and polymorphism of Panax ginseng. Frontiers in Plant Science 5:696-696. 
Figure 1

Gene map of the A. hypogaea chloroplast genomes

Genes shown outside the outer circle are transcribed clockwise and those inside are transcribed counterclockwise. Genes belonging to different functional groups are colorcoded. Dashed area in the inner circle indicates the GC content of the chloroplast genome.

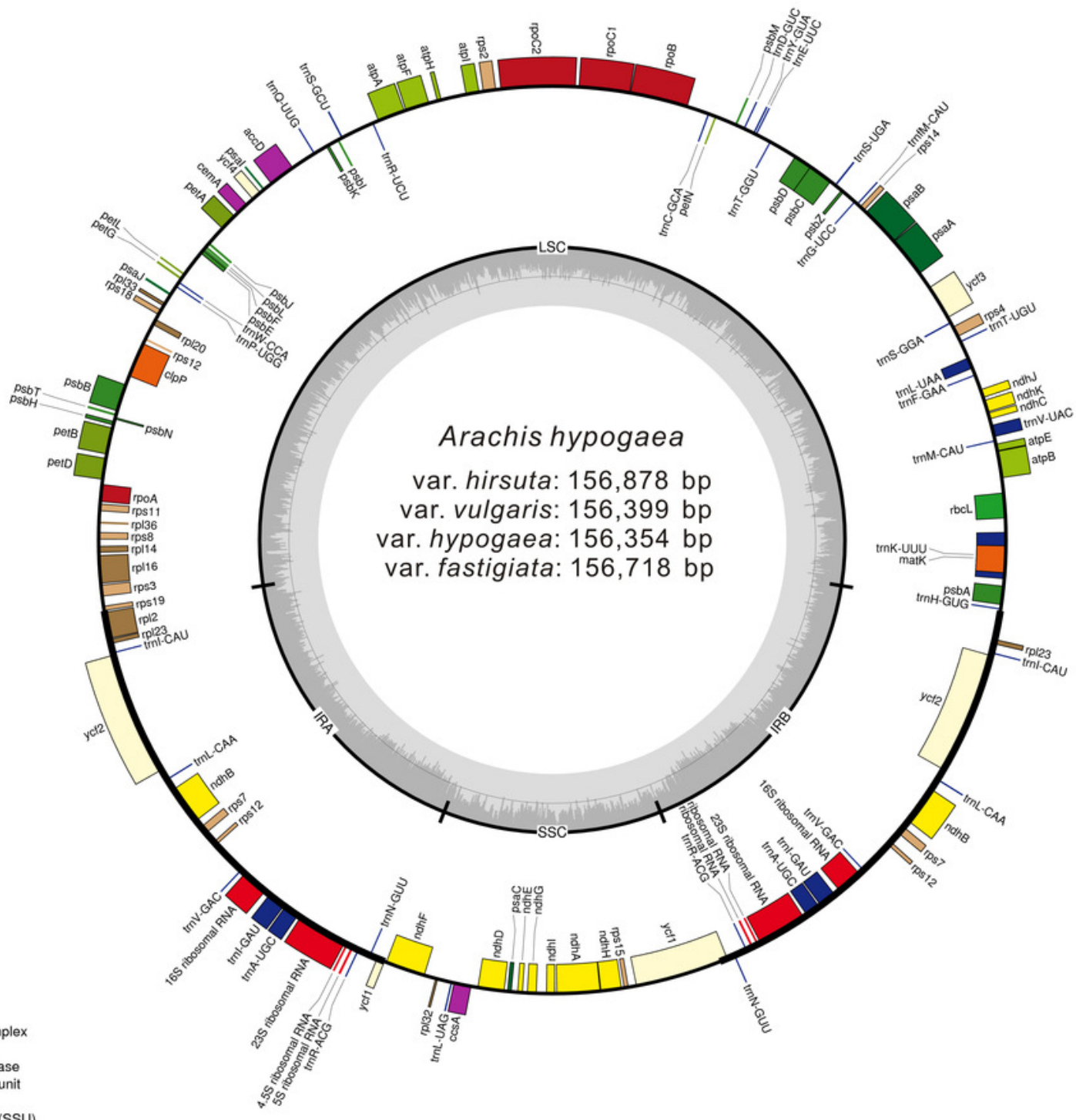


Figure 2

The comparison of the LSC, IR and SSC border regions among the four peanut chloroplast genomes

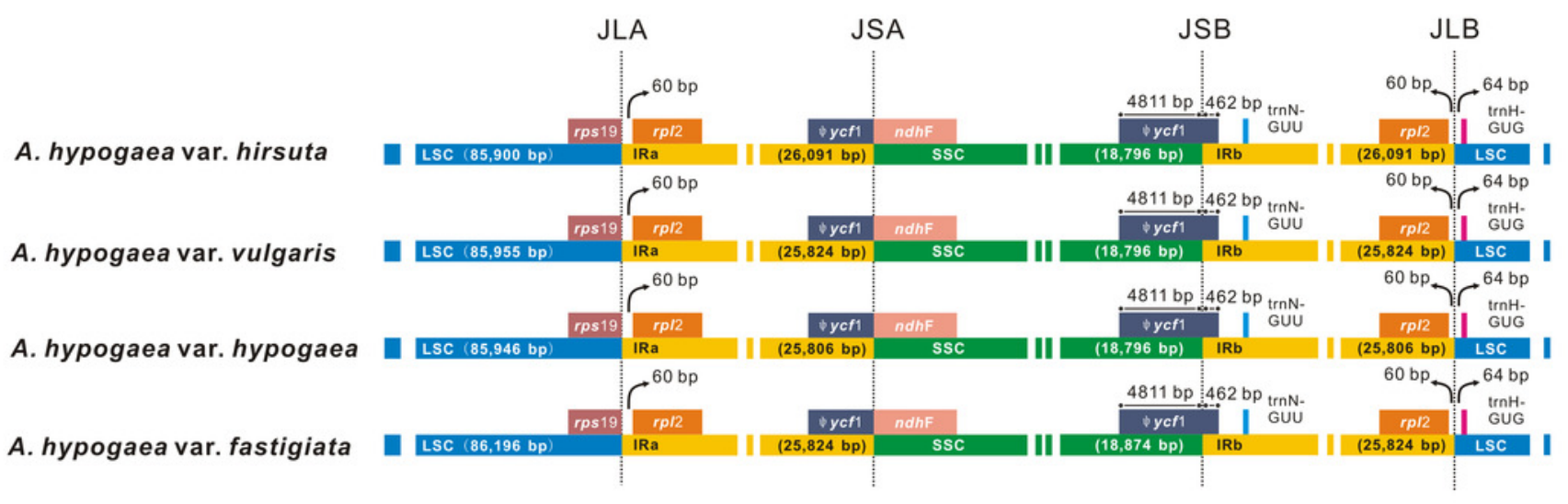




\section{Figure 3}

Visualization of alignment of the peanut chloroplast genome sequences

Genome regions are color-coded as protein coding, rRNA coding, tRNA coding or conserved noncoding sequences (CNS). The $\mathrm{x}$-axis represents the coordinate in the chloroplast genome. Annotated genes are displayed along the top.The sequences similarity of the aligned regions is shown as horizontal bars indicating the average percent identity between $50 \%$ and $100 \%$. 

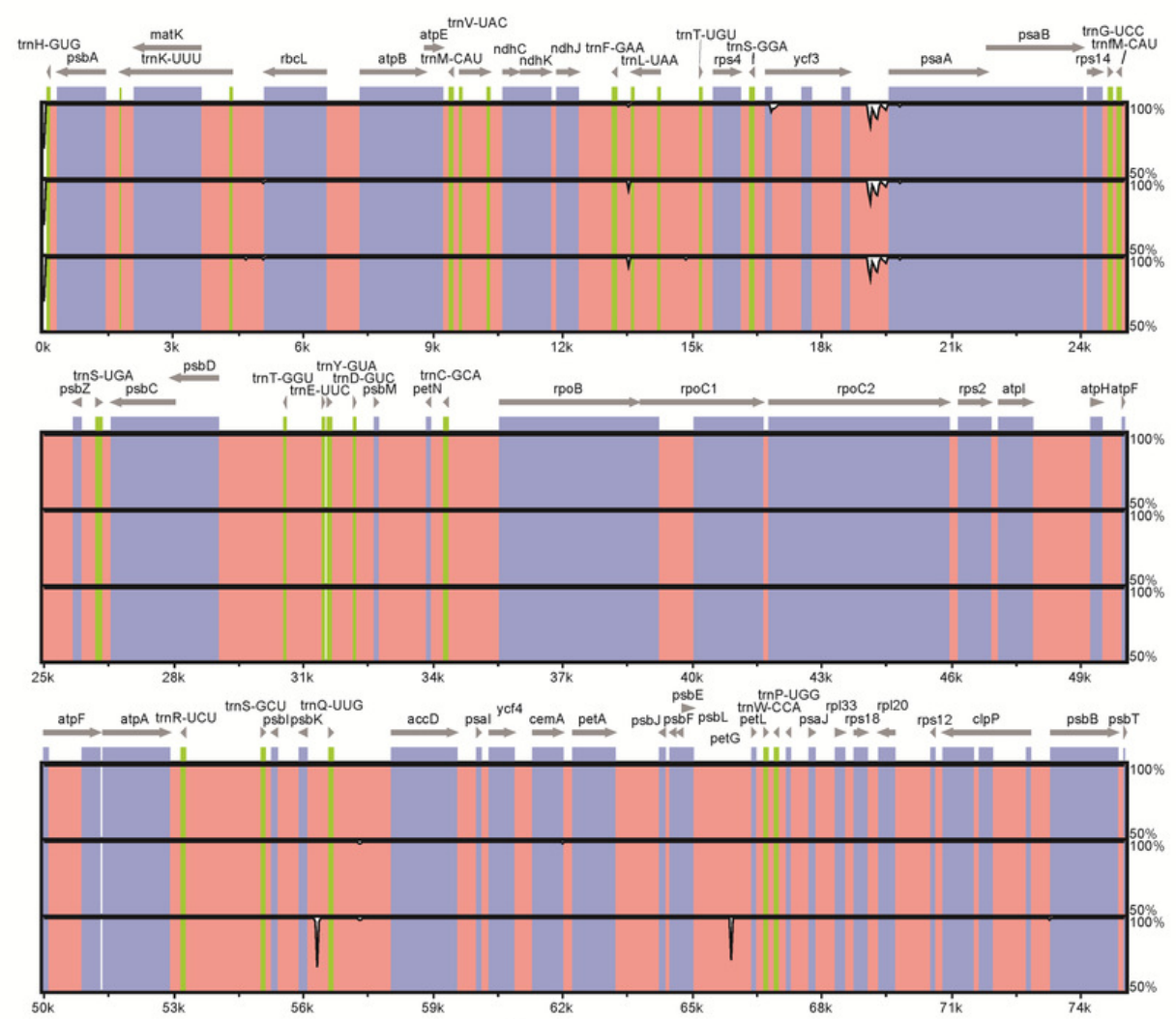

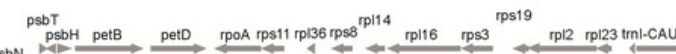

yc12

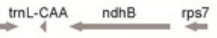

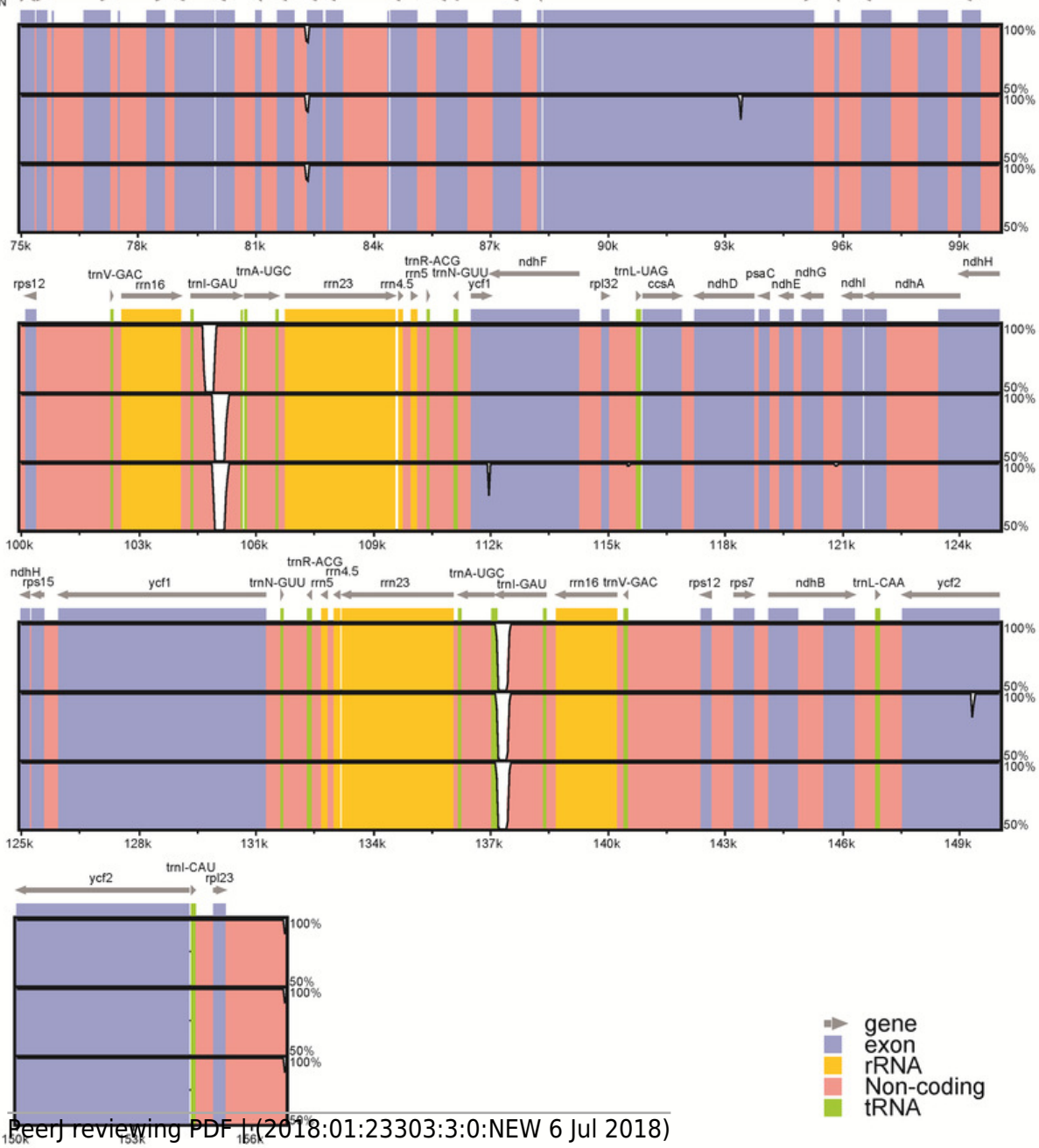


Figure 4

The evolutionary relationship among four cultivated peanuts and the related species of Fabaceae constructed by NJ analyses

Numbers above node are bootstrap support values.

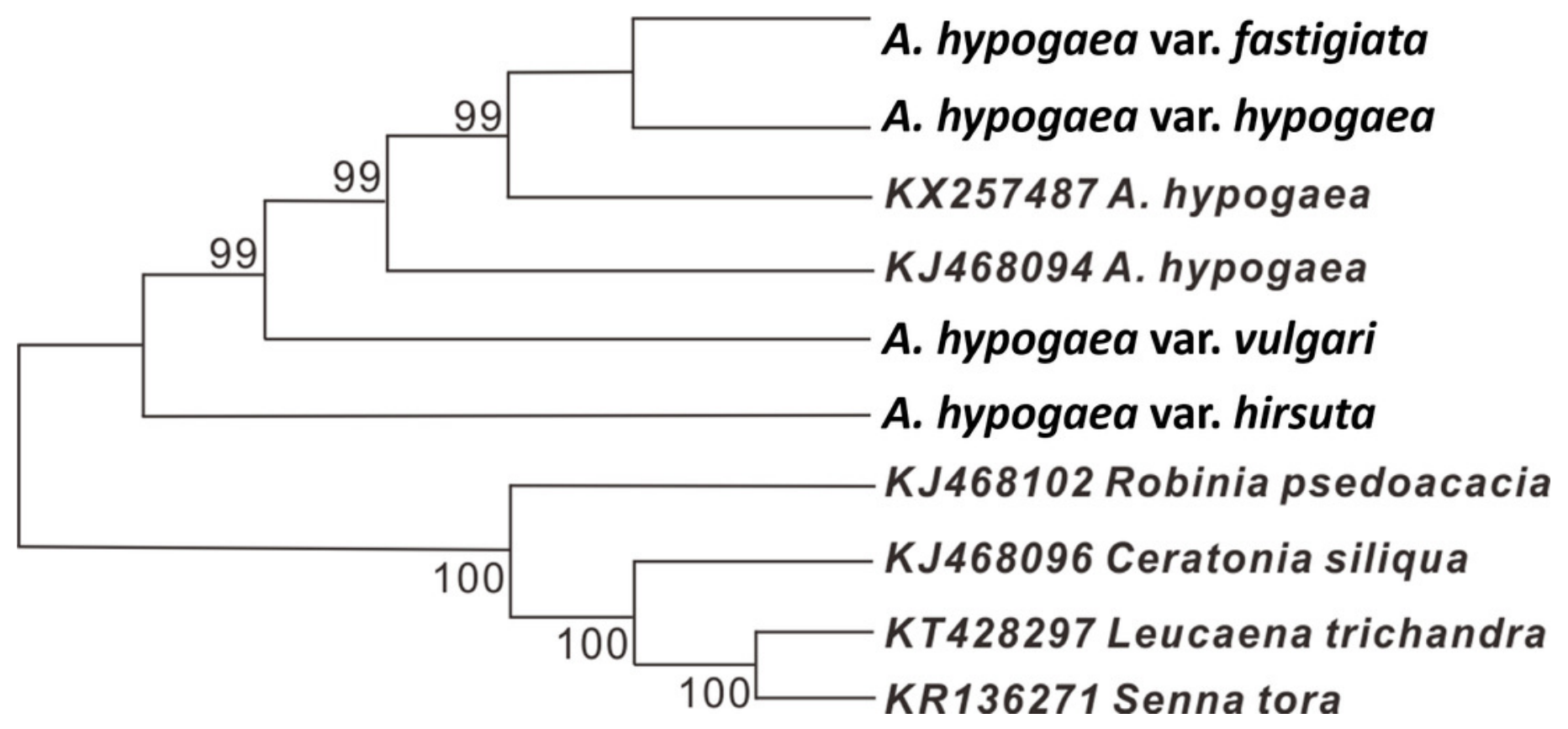




\section{Table $\mathbf{1}$ (on next page)}

Genes identified in the chloroplast genome of peanut

Intron-containing genes are marked by asterisks $(*)$. 
1 Table 1 Genes identified in the chloroplast genome of peanut. Intron-containing genes are marked by asterisks $2(*)$.

\begin{tabular}{|c|c|c|}
\hline Category for genes & Group of genes & Name of genes \\
\hline \multirow[t]{5}{*}{ Self-replication } & tRNA genes & $r r n 5, r r n 4.5, r r n 16, r r n 23$ \\
\hline & rRNA genes & 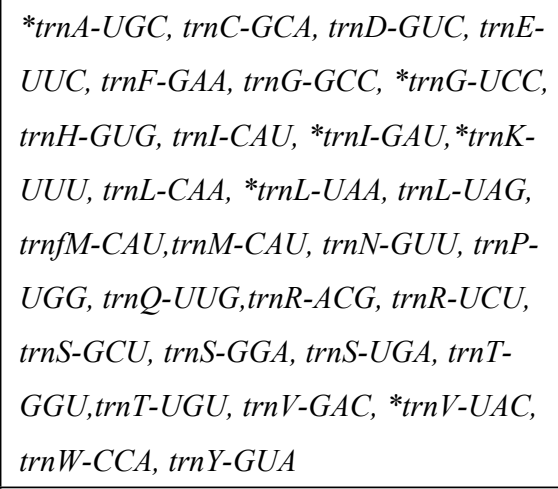 \\
\hline & small subunit of ribosome & $\begin{array}{l}r p s 2, r p s 3, r p s 4, r p s 7, r p s 8, r p s 11, \\
*_{r p s 12, r p s 14, r p s 15,{ }^{*} r p s 16, r p s 18, r p s 19}\end{array}$ \\
\hline & large subunit of ribosome & $\begin{array}{l}\text { rpl2, rpl14, *rpl16, rpl20, rpl22, rpl23, } \\
\text { rpl32, rpl33,rpl36 }\end{array}$ \\
\hline & DNA dependent RNA polymerase & rроA, rров, *rроC1, rроC2 \\
\hline \multirow[t]{6}{*}{ Genes for photosynthesis } & Subunits of NADH-dehydrogenase & $\begin{array}{l}*_{n d h A}{ }^{*} n d h B, n d h C, n d h D, n d h E, \\
n d h F, n d h G, n d h H, n d h I, n d h J, n d h K\end{array}$ \\
\hline & Subunits of photosystem I & $p s a A, p s a B, p s a C, p s a I, p s a J$ \\
\hline & Subunits of photosystem II & $\begin{array}{l}p s b A, p s b B, p s b C, p s b D, p s b E, p s b F, \\
p s b H, p s b I, p s b J, p s b K, p s b L, p s b N, p s b T \text {, } \\
p s b Z\end{array}$ \\
\hline & Subunits of cytochrome $b / f$ complex & petA, *pet $B,{ }^{*}$ pet $D, \operatorname{pet} G, \operatorname{pet} L, \operatorname{pet} N$, \\
\hline & Subunits of ATP synthase & atp $A$, atp $B$, atpE, *atpF, atpH, atpI \\
\hline & Large subunit of rubisco & $r b c L$ \\
\hline \multirow[t]{5}{*}{ Other genes } & Maturase & matK \\
\hline & Protease & $*^{\prime} \operatorname{lp} P$ \\
\hline & Envelope membrane protein & сетA \\
\hline & Subunit of Acetyl-CoA-carboxylase & $a c c D$ \\
\hline & c-type cytochrome synthesis gene & $\operatorname{ccs} A$ \\
\hline Genes of unknown function & Open Reading Frames (ORF, ycf) & $y c f 1, y c f 2, * y c f 3, y c f 4$ \\
\hline
\end{tabular}


Table 2 (on next page)

Details of the complete chloroplast genomes of four peanut botanical varieties 
1 Table 2 Details of the complete chloroplast genomes of four peanut botanical varieties.

2

\begin{tabular}{|l|l|l|l|l|}
\hline & AHL & AHZ & AHP & AHD \\
\hline Matched reads (bp) & $62,087,400$ & $22,511,400$ & $61,928,100$ & $34,570,200$ \\
\hline Genome size (bp) & 156,878 & 156,399 & 156,354 & 156,718 \\
\hline Mean coverage( $\times$ ) & 395.77 & 143.94 & 396.08 & 220.59 \\
\hline LSC length (bp) & 85,900 & 85,955 & 85,946 & 86,196 \\
\hline SSC length (bp) & 18,796 & 18,796 & 18,796 & 18,874 \\
\hline IR length (bp) & 26,091 & 25,824 & 25,806 & 25,824 \\
\hline LSC GC content (\%) & 33.8 & 33.8 & 33.8 & 33.8 \\
\hline SSC GC content (\%) & 42.9 & 42.9 & 42.9 & 42.9 \\
\hline IR GC content (\%) & 30.3 & 30.3 & 30.3 & 30.2 \\
\hline GC content (\%) & 36.4 & 36.4 & 36.4 & 36.3 \\
\hline Total number of genes & 110 & 110 & 110 & 110 \\
\hline Protein coding genes & 76 & 76 & 76 & 76 \\
\hline rRNA & 4 & 4 & 4 & 4 \\
\hline tRNA & 30 & 30 & 30 & 30 \\
\hline
\end{tabular}

